\title{
Contexto social del debate sobre el control natal en Colombia en las décadas de 1960 y 1970: política, medicina y sociedad
}

\author{
The social context of the birth control debate in Colombia in the \\ 1960s and 1970s: politics, medicine and society
}

\section{Ana María Medina Chávez \\ Investigadora independiente. Calle 30\#3A-32 \\ 110311 - Bogotá - Colombia \\ ammedinach@gmail.com}

\begin{abstract}
MEDINA CHÁVEZ, Ana María. Contexto social del debate sobre el control natal en Colombia en las décadas de 1960 y 1970: política, medicina y sociedad. História, Ciências, Saúde - Manguinhos, Rio de Janeiro, v.21, n.4, out.-dez. 2014, p.1467-1473.
\end{abstract}

\section{Resumen}

El artículo busca presentar el contexto y aproximación preliminares necesarios para comprender y abordar el debate sobre el control natal en Colombia en las décadas de 1960 y 1970. Recoge las principales posturas en conflicto en dicho período, y los discursos y lógicas que permearon la llegada de los programas de planificación norteamericanos a América Latina como forma de control político de los movimientos revolucionarios.

Palabras clave: historia; planificación; control natal; Colombia.

\section{Abstract}

The article seeks to present the necessary context and a preliminary approach to understanding and addressing the birth control debate in Colombia in the 1960s and 1970s. It covers the main conflicting positions during that period and the discourses and logics permeating the arrival of North American family planning programs to Latin America as a form of political control of revolutionary movements.

Keywords: history; family planning; birth control; Colombia. 
L as décadas de 1960 y 1970 fueron particularmente dramáticas para la vida de las mujeres en Colombia. Por una parte, estrenaban su condición de ciudadanas, privilegio otorgado por el Gobierno de Rojas Pinilla en 1953; y por el otro, se insertaban masiva y rápidamente en el mercado laboral de un país que anhelaba la industrialización (Arango Gaviria, 1991, 1993). Miles de mujeres, tanto en Colombia como en otros países latinoamericanos, se desplazaron durante las décadas de 1930, 1940 y 1950, del campo a las ciudades como mano de obra para fábricas y como empleadas domésticas (Guy, 1988; Obregón, 2002). Otras tantas ingresaban a nuevas profesiones, pensadas como ideales para la mujer y que les asignaban papeles importantes en el desarrollo del país desde el ámbito de la protección a la familia y la salud (enfermería - trabajo social), pero también desde profesiones liberales como la medicina y el derecho (Cohen, 1973).

El cuerpo femenino y la manera de vivirlo y de relacionarse con él cambiaron gracias a la aparición de la píldora, el dispositivo intrauterino e incluso la aparición en escena de la idea del sexo como posible fuente de placer para la mujer confirmado por los estudios científicos de Kinsey (década de 1950) y Master \& Jhonson en Estados Unidos (década de 1960). El orden sobre el cuerpo femenino comenzaba a resquebrajarse gracias a la mirada de la ciencia: ya no necesariamente limitaba sus funciones a la reproducción, sino que podía además, sentir placer legitimado por experimentos de laboratorio. De la mano de la ciencia y la tecnología, el papel de la mujer en la sociedad sufrió sus más profundos cambios. La mujer tendría acceso a la píldora y al DIU (Dispositivo Intra Uterino), creados en la década de 1960, y que se consolidaron como mecanismos personales e individuales de control natal, frente al uso tradicional del condón por parte de los hombres y generalmente relacionado con el encuentro sexual con "prostitutas" o mujeres por fuera del matrimonio (Bailey, Lopez-Escobar, Alcides, 1973).

Todas estas transformaciones se dieron en el cuerpo individual de la mujer, pero a la vez en sus relaciones sociales y políticas; y éstas a su vez fueron incluidas en dinámicas de poder internacionales, nacionales y locales.

La revolución cubana (1959) marcaría la historia de los demás países Latinoamericanos. Percibida por muchos como un foco de expansión del "mal del comunismo" y como la expresión del desorden y la ruptura; para otros - y en especial para grupos marginados tradicionalmente del poder - sería el puente entre el discurso comunista y la posibilidad de cuestionamiento del orden y la dominación. Cuestionando profundamente el orden social, la tenencia de la tierra y de los medios de producción y la forma de gobierno, el discurso comunista sería adoptado por grupos de trabajadores - entre ellos/as, las mujeres replanteando su posición y participación sobre la propiedad privada y la sociedad de clases.

A nivel nacional, en Colombia, los sucesivos gobiernos de Alberto Lleras Camargo (1958-1962), Guillermo León Valencia (1962-1966) y Carlos Lleras Restrepo (1966-1970) enmarcados todos dentro del Frente Nacional, como pacto de los partidos conservador y liberal para alternarse el poder, buscarían alternativas para alcanzar el "desarrollo" del país. Sería durante el gobierno de Alberto Lleras Camargo que también se plantearían la alianza con Estados Unidos en la lucha contra el comunismo, así como la adopción de políticas internacionales para favorecerla, a través de la Alianza para el Progreso, acuerdo firmado por los países americanos - excepto Cuba - a desarrollarse durante la década de 1960. Esta 
alianza implicaba no sólo el posicionamiento político de los países frente al comunismo, sino también una serie de medidas que buscaban "el desarrollo" y "el bienestar" de las poblaciones, así como una serie de "paquetes de ayuda" por parte de Estados Unidos que serían retirados si las naciones latinoamericanas no llevaban a cabo las reformas sociales y los desarrollos económicos considerados en las cumbres de Bogotá y Punta del Este o si dejaban de sostener los conceptos democráticos enunciados en dichos acuerdos (Einsenhower et al., 1962).

Se pensaba entonces lograr que los países latinoamericanos alcanzaran los niveles de vida norteamericanos o por lo menos se mantuvieran como aliados estables, y para ello era necesario pensar en el futuro y crear mecanismos para alcanzarlo, es decir, planificar. Desde el Estado se planificó sobre la educación, la industrialización, la agricultura, las vías de transporte y comunicaciones. Igualmente, se planificó sobre las poblaciones, su crecimiento y características deseables para el futuro. Se planificó sobre la familia, su conformación ideal, su papel y protección en la sociedad, apareciendo instituciones como el Instituto de Bienestar Familiar. Todos los ámbitos de la vida pública y privada se vieron envueltos en el ejercicio planificador. Se pensó también en planificar la salud, tan necesaria para incrementar la productividad y fomentar el bienestar de las naciones. Para lograr esta meta en particular se requerían medidas como el suministro de agua potable y desagüe, la reducción de la mortalidad de los menores de cinco años, el control de las enfermedades transmisibles, la erradicación de la malaria, el mejoramiento de la nutrición, el perfeccionamiento y formación de profesionales y auxiliares de salud, las mejoras de los servicios básicos de salud a nivel nacional y local, la intensificación de la investigación científica y la utilización de los conocimientos para prevenir y curar enfermedades (Einsenhower et al., 1962).

La aproximación a la salud tenía una explicación primordialmente etio-patiológica y en este sentido, la medicina - como en otros momentos de la historia - tomaría un papel decisivo en la validación, divulgación y sustentación de las nuevas tecnologías y discursos, en este caso en la reconstrucción de los procesos de salud y enfermedad desde los ideales y modelos norteamericanos. La experiencia norteamericana de fines de siglo XIX y principios del siglo XX, a lo largo y ancho de América Latina, había mostrado que el ejercicio de la medicina y de la salud pública eran mecanismos efectivos para lograr propósitos políticos (Quevedo, 2000; Quevedo et al., 2004). Las acciones en salud pública y la ecuación médica se pensaban como estrategias generales para alcanzar el desarrollo de los Estados Unidos, beneficiar sus estrategias comerciales y en el caso particular de las décadas de 1960 y 1970 lograr - bajo la idea del bienestar común - un buen número de aliados para hacerle frente al comunismo.

Igualmente, el discurso demográfico y los resultados de sus investigaciones literalmente inundaron el espacio académico y de discusión, explicando y sustentando las políticas de planificación familiar para América Latina y los demás países del tercer mundo. En el caso de Colombia, se encuentran estudios demográficos tanto para la época como recientes, que abordan el tema del crecimiento demográfico conocido como "la explosión demográfica", como una amenaza social o "la enfermedad de nuestros tiempos", según afirmara Julio César Turbay Ayala (Turbay Ayala, Caradon, 1968), embajador ante los miembros del Consejo de Poblaciones (Population Council) en la ceremonia de presentación de la Declaración de Poblaciones (1966) y su ratificación un año más tarde en donde firmarían presidentes y 
representantes de treinta países del mundo, entre ellos Colombia y República Dominicana como únicos países latinoamericanos.

Para algunos estudios, el crecimiento poblacional se relacionaba con futuros violentos e inciertos. Estas posturas políticas se basaron principalmente en los escritos de Malthus, quien en 1798 publicaría un ensayo sobre los principios de la población, y sobre el cual parte de las políticas de población de las décadas de 1950-1970 se basarían. Este demógrafo británico partía de que una producción de alimentos superior llevaba al crecimiento de la población, mientras que si la población aumentaba demasiado el crecimiento se frenaba, al producirse hambrunas, enfermedades y guerras. En este sentido el aumento de población fue interpretado en las décadas de 1960 y 1970 por muchos como un verdadero peligro para la expansión modernizadora y la industrialización en América Latina.

Igualmente se encuentran textos que cuestionan al malthusianismo, en particular el texto de Consuegra Higgins (1972), con por lo menos tres ediciones desde su impresión original en 1968, que aborda desde una perspectiva marxista el neomalthusianismo como "doctrina del neoimperialismo". Para Consuegra Higgins, las tesis antinatalistas responderían a los intereses de los países económicamente poderosos empeñados en mantener las condiciones del momento y en achacar a los pueblos nativos la culpa de su propia situación a través de tesis raciales, características emocionales o culturales, según las cuales los "subdesarrollados" serían hiper-sensuales y su reproducción debía ser controlada para lograr el equilibrio en la producción. El crecimiento demográfico fue percibido como un peligro para el desarrollo y como posible insumo para futuras revoluciones, y la planificación familiar, como una opción viable. Mientras para muchos el control de la reproducción en sus versiones más biológicas, serviría en el discurso como herramienta ideal para alcanzar el desarrollo por parte de países del tercer mundo; para otros serviría como herramienta de control ideológico para los países del primer mundo.

En el discurso a favor de la planificación, en una lectura inicial de fuentes primarias, el control de nacimientos como "hecho construido" se basaba, además de en datos del Censo de las Américas de 1950, en maneras particulares de representar el "subdesarrollo", la "población", la manera como se unían los latinoamericanos, y en explicaciones de tipo racial como por ejemplo, la alta frecuencia de "uniones libres" ("amancebamiento", "concubinato") en el país, explicada causalmente, como resultado de la existencia de residuos del régimen de esclavitud; y como consecuencia material y moral de la vida en servidumbre, siendo características "la promiscuidad sexual y la inestabilidad de la vida conyugal". Igualmente esta condición se basaba, de acuerdo al autor del informe, en el "analfabetismo", "en la falta de recursos para la celebración del casamiento y en el deseo sexual preponderante" que impedía que las familias ahorraran para lograr el casamiento formal (Mortara, 1964).

Por otro lado, la unión de los discursos médico y demográfico, dentro de un amplio debate político, junto con las intenciones reformistas de la educación superior, acercarían aún más a la medicina nacional a la órbita y modelo de educación médica norteamericano. Ya desde fines de la década de 1940, con la visita de las misiones médicas norteamericanas en 1948, la misión médica de Lapham en 1953 y la de Tulane en 1957, se plantearon una serie de reformas educativas en las facultades de medicina (como se hacía en las demás facultades), que incluían reformas curriculares basadas en los modelos del norte. En 1958 
se funda Asociación Colombiana de Universidades (ASCUN), y en 1959 aparece Asociación Colombiana de Facultades Médicas (ASCOFAME), como parte de ese ejercicio planificador y organizador de la educación médica (Miranda Canal, Quevedo, Hernandez, 1999). Colombia serviría a partir de la década de 1960 como piloto para proyectos de reforma educativa en toda Latinoamérica, auspiciados por la Organización Panamericana de la Salud, el Memorial Milkbank Fund de Nueva York (Zschock, 1970) y programas y proyectos financiados por instituciones internacionales como el Fondo de Poblaciones - financiado a su vez por la Fundación Rockefeller -, la Internacional Planned Parenthood Association y la Fundación Kellog, entre otros.

La década de 1960 se constituyó en un período de intenso debate, formulación de propuestas y discusiones en el campo de la educación médica: ¿Cómo era el médico que Colombia necesitaba? Y si la misión médica hacía parte constituyente de los proyectos de desarrollo económico de los países "atrasados", ¿qué y cómo debía enseñarse? Dentro de dichas reformas y planes se incluiría la planificación familiar y en particular el control natal como tema central de interés para el médico y los demás profesionales de salud, basados en los resultados demográficos y en el interés por lograr el "desarrollo" (Mendoza Hoyos, 1966, 1968).

Sin embargo, la planificación familiar, el uso de métodos anticonceptivos y la posibilidad de espaciar los embarazos planteaba serias confrontaciones con el pensamiento católico conservador de la época (Perez Ramirez, 1965; Vergara Delgado, 1967; Shea et al., 1971; Simmons, Cardona, 1974), como también encuentros entre los grupos que incorporaban el discurso marxista frente a los nacionalistas, entre ellos el dr. Hernán Vergara, psiquiatra y profesor de psicología que afirmaba que las acciones de planificación tenían como fin los intereses particulares de algunos gobernantes, que de una u otra manera funcionaban como intermediarios de las fundaciones como la Rockefeller (Stycos, 1968; Vergara Delgado, 1968)

Preguntas como: ¿Podía el matrimonio no conllevar a la procreación? ¿Podían las relaciones sexuales no tener como fin último la reproducción sino el placer? ¿Podía el crecimiento de la población controlarse a favor del "desarrollo"? Y es tal vez en esta encrucijada entre el ideal de desarrollo y el pensamiento católico, que la ciencia surge como posible respuesta: el discurso médico, junto al discurso demográfico, se enlaza con los discursos desarrollistas de la época.

Al parecer, la opción del gobierno frente al conflicto que producía discutir y planificar los nacimientos, fue introducir a partir de 1969 un plan de desarrollo que incluyó un conjunto de propuestas para la planificación familiar dentro de los programas de salud materna e infantil, pero sin especificar objetivos demográficos. Formulando una política pública legitimó y estimuló la prestación de servicios por parte del sector privado (Amadeo, Chernichovsky, Ojeda, 1991). Fue así como en los anõs 1960 una institución privada se encargó de desarrollar los programas de planificación familiar. La Asociación Pro Bienestar de la Familia (PROFAMILIA) fue fundada en 1965, funcionando como un consultorio privado del médico Fernando Tamayo, dirigido a familias con escasos recursos económicos. En 1966 se afilia a la Internacional Planned Parenthood Foundation (regional del hemisferio occidental), y en ese año funda su clínica piloto en Bogotá. Establece en los años siguientes una red de trabajo con 42 clínicas y centros de planificación familiar. La esterilización voluntaria se inicia en 1970 con el programa de vasectomía y la femenina en 1971, año en que inaugura su 
programa de distribución basado en la comunidad, dirigido a comunidades locales. Incluye un programa de Información, Educación y Comunicación, que incluye elaboración de panfletos, manuales de entrenamiento, posters, radio y conferencias. El programa de distribución basado en la comunidad se inaugura en 1971 en comunidades rurales como parte de un convenio con la Asociación Nacional de Cafeteros. Luego, se crearía un programa urbano en 1974 (Amadeo, Chernichovsky, Ojeda, 1991). Colombia sería entonces uno de los primeros países latinoamericanos en pensar, planificar y aplicar programas de control natal en el marco de la búsqueda por un Estado de bienestar, no como política explícita y activa del Estado sino a través de entidades de carácter privado y dentro de un marco de intereses internacionales.

Es así como los programas de control natal elaborados y ejecutados en Colombia le permitieron en la década de 1970 - a consideración de los investigadores - ser uno de los pocos países en Latino América en lograr un descenso dramático del crecimiento de la población (ASCOFAME, 1975; Carvajal, Geithman, 1976; Comité..., 1977), registrándose durante los años siguientes un descenso en el promedio de hijos vivos por mujer de 7 en 1964 a 3,9 en 1978, sintiéndose el descenso con especial intensidad en el área urbana (Puyana, 1985).

Pero, ¿se trataba de un interés colectivo y generalizado en la sociedad? ¿O se trataba del interés de grupos dominantes tanto nacionales como internacionales utilizando los "discursos científicos de la medicina y la demografía" para legitimar sus intereses de clase? ¿Qué papel cumplieron los diversos actores tanto individuales como institucionales y de qué manera construían y representaban el mundo reproductivo? ¿Cómo vivieron y asumieron el conflicto las mujeres de la época? ¿Qué implicaciones tuvo para ellas como sujetos socio-políticos?

Como se ve, el control natal, tanto como conocimiento científico y como representación social, se configura dentro de un campo de fuerzas y luchas por el poder, más allá de la aplicación de técnicas reproductivas neutras. Más que discutir la efectividad o no de una determinada técnica o proceso biomédico, es importante que discutamos los mecanismos por medio de los cuales llegamos a considerarlos correctos, verdaderos o legítimos de aplicar.

El campo de la salud reproductiva, desde una perspectiva histórica, puede brindar nuevas e interesantes perspectivas, tanto en la teoría como en la práctica y en la generación de políticas públicas. Igualmente, explorar en profundidad estas preguntas, permitirá la reflexión crítica sobre el cuerpo, las formas de ejercer políticamente sobre él, y la manera cómo las tecnologías son incorporadas de manera naturalizada.

\section{REFERENCIAS}

AMADEO, Jesus; CHERNICHOVSKY, Dov; OJEDA, Gabriel.

The profamilia family planning program, Colombia: an economic perspective. Washington, D.C.: World Bank. 1991.

ARANGO GAVIRIA, Luz Gabriela.

Mujeres obreras, familia y políticas empresariales: la historia de Fabricato. Bogotá: Universidad de los Andes. 1993.

ARANGO GAVIRIA, Luz Gabriela.

Mujer, religión e industria: Fabricato, 1923-1982.
Medellín: Universidad de Antioquia; Universidad Externado de Colombia. 1991.

ASCOFAME.

Asociación Colombiana de Facultades de Medicina. Informes de población/planificación familiar. Bogotá: Asociación Colombiana de Facultades de Medicina. División de estudios de población 1969-1975. 1975.

BAILEY, Jerald; LOPEZ-ESCOBAR, Guillermo; ALCIDES, Estrada.

A Colombian view of the condom. Studies in Family Planning, v.4, n.3, p.60-64. 1973. 
CARVAJAL, Manuel; GEITHMAN, David. Family planning and family size determination: the evidence from seven Latin American cities. Gainesville: University Press of Florida. 1976.

COHEN, Lucy.

Woman's entry to the professions in Colombia: Selected characteristics. Journal of Marriage and the family, v.35, n.2, p.322-330. 1973.

COMITÉ...

Comité de Trabajo para el Estudio de Impacto de la Planificación Familiar Sobre la Estructura Demográfica, Económica y Social de Colombia. Descenso de la fecundidad y planificación familiar en Colombia 1964-1975. Bogotá: Corporación Centro Regional de Población. 1977.

CONSUEGRA HIGGINS, José.

El control de la natalidad como arma del imperialismo. Barranquilla: Eds. de la Universidad del Atlántico. 1972.

EINSENHOWER, Milton et al.

La alianza para el progreso. México: Editorial Novaro-México. 1962.

\section{GUY, Donna.}

White slavery, public health, and the socialist position on legalized prostitution in Argentina, 1913-1936. Latin American Research Review, v.23, n.3, p.60-80. 1988.

MENDOZA HOYOS, Hernán.

The Colombian program for public education, personnel training and evaluation. Demography, v.5, n.2, p.827-835. 1968.

MENDOZA HOYOS, Hernán.

Aspectos médicos de la planificación familiar. Bogotá: Asociación de Facultades de Medicina. 1966.

MIRANDA CANAL, Nestor; QUEVEDO, Emilio; HERNANDEZ, Mario (Ed.).

Historia social de la ciencia en Colombia: la institucionalización de la medicina en Colombia. tomo 8. Bogotá: COLCIENCIAS. 1999.

MORTARA, Giorgio.

Características de la estructura demográfica de los países americanos. Washington, D.C.: Organización de los Estados Americanos. 1964.

OBREGÓN, Diana.

Médicos, prostitución y enfermedades venéreas en Colombia, 1886-1951. História, Ciências, Saúde - Manguinhos, v.9, supl., p.161-186. 2002.
PEREZ RAMIREZ, Gustavo.

La Iglesia Católica y la planificación familiar: perspectivas en 1965. Bogotá: Asociación Colombiana Para el Estudio Científico de la Población. 1965.

PUYANA, Yolanda.

Mujer y familia en Colombia. In: Bonilla, Elssy. Mujer y familia en Colombia. Bogotá: Plaza y Janés. p.177-204. 1985.

QUEVEDO, Emilio.

El tránsito desde la higiene hacia la salud pública en América Latina. Tierra Firme, v.72, n.18, p.611-661. 2000.

QUEVEDO, Emilio et al.

Café y gusanos, mosquitos y petróleo: el tránsito desde la higiene hacia la medicina tropical y la salud pública en Colombia, 1873-1953. Bogotá: Universidad Nacional de Colombia. 2004.

SHEA, Gail A. et al.

Catholic parish priest and birth control: a comparative study of opinion in Colombia, the United States and the Netherlands. Studies in Family Planning, v.2, n.6, p.121-136. 1971.

SIMMONS, Allan; CARDONA, Ricardo.

Colombia: stages of family planning adoption, 1964-1969. Studies in Family Planning, v.5, n.2, p.42-49. 1974.

STYCOS, Joseph Mayone.

Opposition to family planning in Latin America. Demography, v.5, n.2, p.846-854. 1968.

TURBAY AYALA, Julio Cesar; CARADON, Lord. Declaration on population: the world leaders statement. Studies in Family Planning, v.1, n.26, p.1-3. 1968 .

VERGARA DELGADO, Hernan. El complejo de Layo: antecedentes e interrogantes de la política demográfica. v.1. Bogotá: Tercer Mundo. 1968.

VERGARA DELGADO, Hernan.

La planificación familiar: desafío de la iglesia. Revista Javeriana, v.67, n.332, p.185-228. 1967.

ZSCHOCK, Dieter D.

Health planning in Latin America: review and evaluation. New York: Milkbank Memorial Fund. 1970.

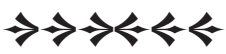

